\title{
Lichen flora of Rodnei Mountains National Park (Eastern Carpathians, Romania) including new records for the Romanian mycoflora
}

\author{
Ioana Violeta Ardelean ${ }^{1,2}$, Christine Keller ${ }^{1} \&$ Christoph Scheidegger ${ }^{1}$ \\ ${ }^{1}$ WSL, Swiss Federal Research Institute, 8903 Birmensdorf, Switzerland. \\ E-mail: ioana.ardelean@wsl.ch \\ ${ }^{2}$ Faculty of Biology and Geology, Babeș-Bolyai University, Cluj-Napoca, Romania
}

\begin{abstract}
We investigated the lichen flora of the main habitats existing in Rodnei Mountains identifying 283 lichen species, and one subspecific taxon. Of these, 67 taxa are new records for the lichen flora of Romania, and 182 species are reported for the first time in Rodnei Mountains. Considering previous reports and our results, 442 lichen taxa are reported in Rodnei Mountains region in total, accounting for approx. $35 \%$ of the total lichen flora of Romania. When comparing the Red Lists of Romania and surrounding Carpathian countries, our data revealed the presence of a high number of threatened species in the region.
\end{abstract}

\section{INTRODUCTION}

During the last years, various lichenological studies reported new species for the Romanian lichen flora (Crișan, 2006; Çobanoğlu et al., 2009, 2011; Yavuz \& Çobanolu, 2008; Vondrák $\&$ Šoun, 2008), but at the same time, species that had been recorded before, such as Anzina carneonivea (Scheidegger, 1985) and Lepraria incana (Bartók, 1999) are missing in the current checklist (Ciurchea, 2004).

260 lichen species from 11 locations were previously reported in Rodnei Mountains (Ciurchea, 2004), and reflected the high species richness of the national park. However, the knowledge of lichen species distribution is still scattered in Romania and some of the species records have not been rechecked since more than a century (Bartok $\&$ Crișan, personal communication).

Conserved areas are well known sanctuaries for threatened species including various groups of lichens (Goward, 1995; Zoller et al., 2000; Nascimbene et al., 2013; Ignatov et al., 2004; Lackovičová \& Guttová, 2006). Furthermore, lichen habitats such as old-growth forest stands and veteran trees in extensively managed meadows with their important lichen microhabitats are often lost in managed and perturbed areas (Wolseley, 1995; Thor, 1995; Scheidegger \& Werth, 2009).

The aim of this study was to assess the lichen flora of characteristic habitats of the Rodnei
Mountains in a replicated design with a standardized lichen diversity assessment. This study should also reveal the importance of conserved areas for the maintenance of lichen diversity in the Rodnei Mountains and thus contribute to decisions about future conservation strategies within this biosphere reserve.

\section{MATERIALS AND METHODS}

\section{Study area}

The Rodnei Mountains are located in the northern part of the Eastern Carpathians reaching their highest elevation at Pietrosul Mare Peak $(2303 \mathrm{~m})$. Most of the study area is part of the Rodnei Mountains National Park, established in 1932 and declared as a UNESCO Biosphere Reserve in 1979.

\section{Climate}

Due to the position and orientation (East-West) of the mountains, the climate is characterised by the Baltic and the Oceanic influences. The mean annual temperature decreases with altitude, ranging between $6{ }^{\circ} \mathrm{C}$ at the base of the mountains and $-1.5{ }^{\circ} \mathrm{C}$ at the highest altitudes. Mean annual precipitations range from 1300 to 1400 mm (Gorduza, 1983).

\section{Sampling}

The sampling method was structured according to the sampling design described by Scheidegger 
et al. (2002). Four main substrates were considered in each circular plot of 1 ha: trees, dead wood, soil and rock, and for each we applied 6 relevés. If the substrate was not available or it was not colonised with lichens, it was substitued with other available substrates, thus achieving 24 relevés in each plot. All lichen species within a relevé surface of $0.2 \mathrm{~m}^{2}$ were collected (except the crustose lichens from rocks).

The investigated habitats are well represented in the Rodnei Mountains and follow an altitudinal gradient from wooded meadows to mixed and coniferous forests, to Pinus mugo shrubs and alpine vegetation with bare rocks. Each of the five habitats was analysed at two levels: conserved and managed (except Pinus mugo shrubs and wooded meadows, for which only one type of management was found in the study area). There were seven replicated circular plots of 1 ha for each habitat with its corresponding levels of conservation (i.e., 56 plots in total). The minimum distance between the plots was $100 \mathrm{~m}$. The selection of the 9 sampling localities (Fig. 1) aimed at covering the types of habitat and stand characteristics (Table 1) of the investigated area.

The lichen specimens were identified based on morphological and chemical characteristics using mostly the keys of Smith et al. (2009), Wirth (1995) and Tønsberg (1992).

For the crustose sterile species and the specimens that needed chemical analyses for identification, thin layer chromatography (TLC) was used according to the methods described in White \& James (1985) with solvents A, B and C.

The specimens are stored in the Herbaria of "Alexandru Borza" Botanical Garden, BabeșBolyai University, Cluj-Napoca, Romania.

\section{Data assessment}

The importance of lichen flora in our results was determined by the total number of species, the number of new species for the region, and the new species for Romanian lichen flora. We also took into account the number of species in the Red List of macrolichens from Romania (Bartok \& Crișan, personal communication)

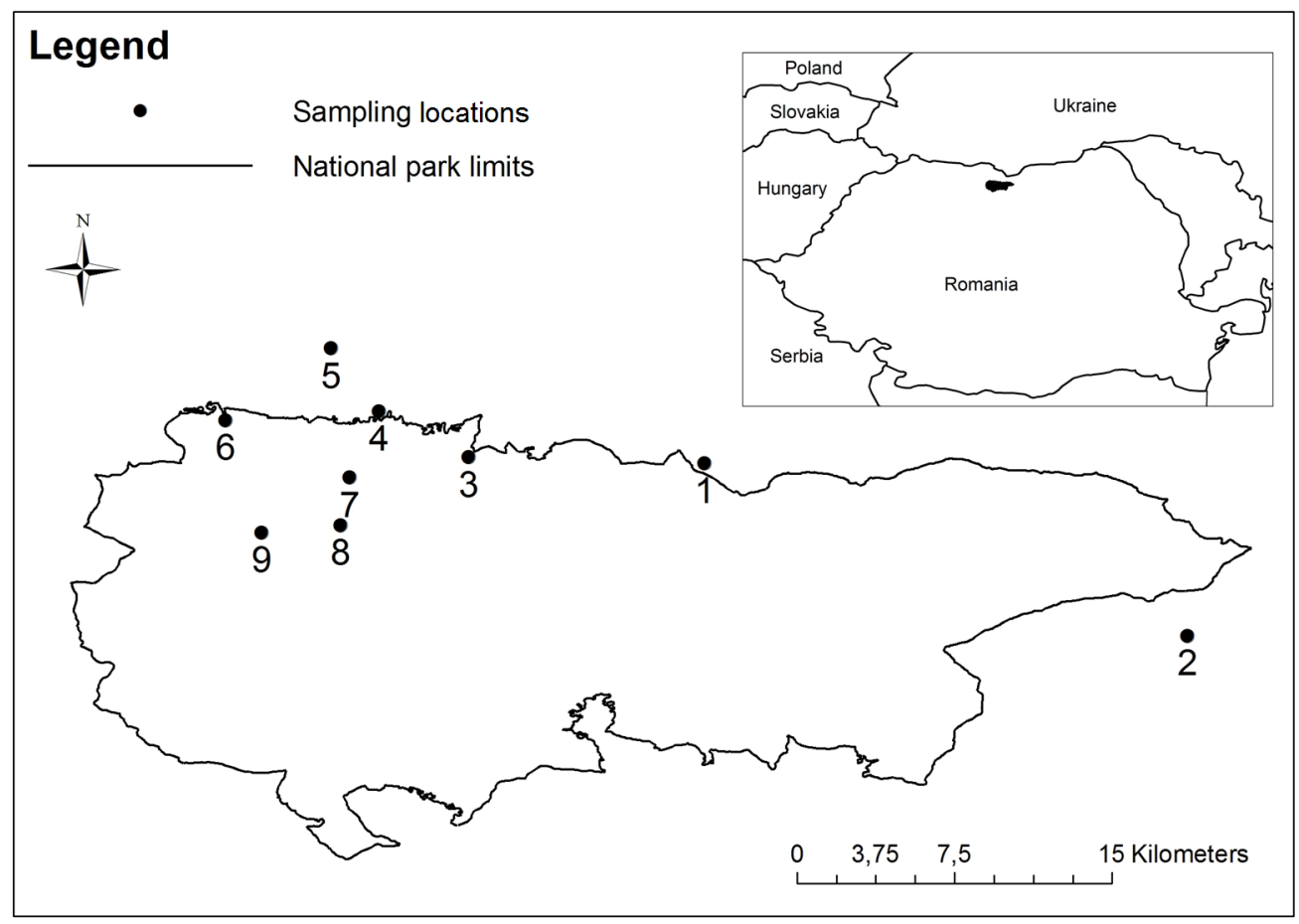

Fig. 1. Location of the Rodnei Mts National Park in Romania, and study area with sampling localities. 1 - Cascada Cailor, 2 - Rotunda Pass, 3 - Repedea Valley, 4 - Borșa 1, 5 - Borşa 2, 6 - Izvorul Dragoș Valley, 7 - Pietrosul Mare, 8 - Gropile, 9 - Bătrâna. 
Table 1. 56 investigated plots (within the 9 sampling localities, see Fig. 1). Abbreviations: AC conserved alpine vegetation with bare rocks, AM - managed alpine vegetation with bare rocks, $\mathrm{PM}$ - Pinus mugo shrubs, CC - conserved coniferous forest, CM - managed coniferous forest, MC - conserved mixed forest, MM - managed mixed forest, WM - wooded meadows, C - conserved, $\mathrm{M}$ - managed.

\begin{tabular}{|c|c|c|c|c|c|c|c|c|}
\hline Plot code & Location name & Coordinates $\mathrm{x}$ & Coordinates y & Altitude (m) & Exposition & Slope $\left[{ }^{\circ}\right]$ & $\begin{array}{c}\text { Conservation } \\
\text { status }\end{array}$ & Habitat type \\
\hline $\mathrm{AC} 1$ & Pietrosul Mare & 47,5941 & 24,6365 & 2143 & W & 33 & $\mathrm{C}$ & alpine vegetation \\
\hline AC2 & Pietrosul Mare & 47,5915 & 24,6379 & 2125 & $\mathrm{E}$ & 10 & $\mathrm{C}$ & alpine vegetation \\
\hline AC3 & Pietrosul Mare & 47,5955 & 24,6505 & 2136 & E & 5 & $\mathrm{C}$ & alpine vegetation \\
\hline AC4 & Pietrosul Mare & 47,5935 & 24,6407 & 2171 & S & 39 & $\mathrm{C}$ & alpine vegetation \\
\hline AC5 & Pietrosul Mare & 47,6004 & 24,6231 & 1916 & W & 39 & $\mathrm{C}$ & alpine vegetation \\
\hline AC6 & Pietrosul Mare & 47,5994 & 24,6304 & 2193 & S & 12 & $\mathrm{C}$ & alpine vegetation \\
\hline AC7 & Pietrosul Mare & 47,5996 & 24,6279 & 2173 & S & 43 & $\mathrm{C}$ & alpine vegetation \\
\hline AM1 & Gropile & 47,579 & 24,6325 & 2085 & E & 7 & M & alpine vegetation \\
\hline AM2 & Gropile & 47,5703 & 24,6468 & 2020 & $S$ & 24 & M & alpine vegetation \\
\hline AM3 & Gropile & 47,5715 & 24,6396 & 2004 & $S$ & 5 & M & alpine vegetation \\
\hline AM4 & Gropile & 47,5739 & 24,6344 & 1085 & $S$ & 29 & M & alpine vegetation \\
\hline AM5 & Bătrâna & 47,5699 & 24,6124 & 1915 & $S$ & 24 & M & alpine vegetation \\
\hline AM6 & Bătrâna & 47,568 & 24,6116 & 1816 & S & 26 & M & alpine vegetation \\
\hline AM7 & Bătrâna & 47,5782 & 24,6032 & 1719 & W & 24 & M & alpine vegetation \\
\hline PC1 & Pietrosul Mare & 47,6018 & 24,6479 & 1785 & $\mathrm{E}$ & 14 & $\mathrm{C}$ & Pinus mugo shrubs \\
\hline PC2 & Pietrosul Mare & 47,5886 & 24,6472 & 1882 & S & 36 & $\mathrm{C}$ & Pinus mugo shrubs \\
\hline PC3 & Pietrosul Mare & 47,6019 & 24,6506 & 1788 & $\mathrm{~N}$ & 28 & $\mathrm{C}$ & Pinus mugo shrubs \\
\hline PC4 & Pietrosul Mare & 47,5913 & 24,6437 & 2040 & $S$ & 32 & $\mathrm{C}$ & Pinus mugo shrubs \\
\hline PC5 & Pietrosul Mare & 47,6014 & 24,6229 & 2019 & W & 33 & $\mathrm{C}$ & Pinus mugo shrubs \\
\hline PC6 & Pietrosul Mare & 47,6071 & 24,6113 & 1821 & W & 26 & $\mathrm{C}$ & Pinus mugo shrubs \\
\hline PC7 & Bătrâna & 47,5692 & 24,596 & 1781 & S & 30 & $\mathrm{C}$ & Pinus mugo shrubs \\
\hline $\mathrm{CC} 1$ & Repedea Valley & 47,5964 & 24,6918 & 1164 & $\mathrm{E}$ & 26 & $\mathrm{C}$ & spruce forest \\
\hline $\mathrm{CC} 2$ & Repedea Valley & 47,5952 & 24,679 & 1326 & $S$ & 38 & $\mathrm{C}$ & spruce forest \\
\hline CC3 & Pietrosul Mare & 47,6059 & 24,6067 & 1596 & $S$ & 32 & $\mathrm{C}$ & spruce forest \\
\hline $\mathrm{CC} 4$ & Pietrosul Mare & 47,6028 & 24,6054 & 1477 & S & 33 & $\mathrm{C}$ & spruce forest \\
\hline CC5 & Pietrosul Mare & 47,6053 & 24,6026 & 1470 & S & 37 & $\mathrm{C}$ & spruce forest \\
\hline CC6 & Pietrosul Mare & 47,6036 & 24,6002 & 1353 & W & 25 & $\mathrm{C}$ & spruce forest \\
\hline CC7 & Pietrosul Mare & 47,6014 & 24,6013 & 1316 & S & 37 & $\mathrm{C}$ & spruce forest \\
\hline CM1 & Borșa1 & 47,62 & 24,6589 & 1148 & $\mathrm{~N}$ & 27 & M & spruce forest \\
\hline $\mathrm{CM} 2$ & Repedea Valley & 47,5938 & 24,7012 & 1151 & $\mathrm{~N}$ & 31 & M & spruce forest \\
\hline CM3 & Repedea Valley & 47,5982 & 24,7051 & 1375 & W & 36 & M & spruce forest \\
\hline CM4 & Repedea Valley & 47,6018 & 24,7087 & 1357 & $\mathrm{~N}$ & 25 & M & spruce forest \\
\hline CM5 & Cascada Cailor & 47,6062 & 24,795 & 1275 & W & 14 & M & spruce forest \\
\hline CM6 & Izvorul Dragoș Valley & 47,5982 & 24,6022 & 1081 & $\mathrm{E}$ & 35 & M & spruce forest \\
\hline CM7 & Izvorul Dragoș Valley & 47,5989 & 24,6016 & 932 & $\mathrm{~N}$ & 19 & M & spruce forest \\
\hline $\mathrm{MC1}$ & Repedea Valley & 47,5972 & 24,6907 & 966 & $\mathrm{~N}$ & 40 & $\mathrm{C}$ & mixed forest \\
\hline MC2 & Cascada Cailor & 47,5951 & 24,797 & 1207 & $\mathrm{~N}$ & 35 & $\mathrm{C}$ & mixed forest \\
\hline MC3 & Rotunda Pass & 47,5244 & 24,9998 & 1044 & W & 21 & $\mathrm{C}$ & mixed forest \\
\hline MC4 & Rotunda Pass & 47,5284 & 25,0041 & 1089 & W & 14 & $\mathrm{C}$ & mixed forest \\
\hline MC5 & Izvorul Dragoș Valley & 47,6083 & 24,5874 & 863 & $\mathrm{E}$ & 35 & $\mathrm{C}$ & mixed forest \\
\hline MC6 & Repedea Valley & 47,5943 & 24,6815 & 1180 & $\mathrm{E}$ & 44 & $\mathrm{C}$ & mixed forest \\
\hline MC7 & Izvorul Dragoș Valley & 47,6016 & 24,5935 & 1019 & $\mathrm{~N}$ & 15 & $\mathrm{C}$ & mixed forest \\
\hline MM1 & Repedea Valley & 47,5899 & 24,7002 & 1196 & W & 42 & M & mixed forest \\
\hline MM2 & Repedea Valley & 47,6149 & 24,6977 & 866 & W & 14 & M & mixed forest \\
\hline
\end{tabular}


Table 1 (continued)

\begin{tabular}{|c|c|c|c|c|c|c|c|c|}
\hline Plot code & Location name & Coordinates $\mathrm{x}$ & Coordinates y & Altitude (m) & Exposition & Slope $\left[^{\circ}\right]$ & $\begin{array}{c}\text { Conservation } \\
\text { status }\end{array}$ & Habitat type \\
\hline MM3 & Repedea Valley & 47,6128 & 24,6871 & 1257 & $\mathrm{E}$ & 35 & M & mixed forest \\
\hline MM4 & Repedea Valley & 47,6146 & 24,6922 & 1058 & $\mathrm{E}$ & 33 & M & mixed forest \\
\hline MM5 & Izvorul Dragoș Valley & 47,6227 & 24,5758 & 903 & $\mathrm{~N}$ & 32 & M & mixed forest \\
\hline MM6 & Izvorul Dragoș Valley & 47,6228 & 24,5858 & 930 & $\mathrm{~N}$ & 41 & M & mixed forest \\
\hline MM7 & Izvorul Dragoș Valley & 47,6242 & 24,5957 & 915 & $S$ & 5 & M & mixed forest \\
\hline WM1 & Borșa1 & 47,6216 & 24,6494 & 1095 & $\mathrm{~N}$ & 18 & M & wooded meadows \\
\hline WM2 & Borșa1 & 47,6224 & 24,6396 & 1048 & $\mathrm{~N}$ & 13 & M & wooded meadows \\
\hline WM3 & Borșa1 & 47,6228 & 24,6325 & 1048 & $\mathrm{~N}$ & 6 & M & wooded meadows \\
\hline WM4 & Borșa1 & 47,6287 & 24,6635 & 1988 & $\mathrm{~N}$ & 16 & M & wooded meadows \\
\hline WM5 & Borșa2 & 47,6504 & 24,6356 & 783 & $\mathrm{~N}$ & 10 & M & wooded meadows \\
\hline WM6 & Izvorul Dragoș Valley & 47,6209 & 24,6176 & 1036 & $\mathrm{~N}$ & 23 & M & wooded meadows \\
\hline WM7 & Izvorul Dragoș Valley & 47,6211 & 24,6125 & 957 & $\mathrm{~N}$ & 33 & M & wooded meadows \\
\hline
\end{tabular}

and the Red Lists of the surrounding countries which harbour the Carpathian mountain ridge: Hungary (Lőkös \& Tóth, 1996), Ukraine (Didukh, 2009), Slovakia (Pisút et al., 2001), and Poland (Cieslinski et al., 2003), with detailed information in Table 2.

\section{RESULTS AND DISCUSSIONS}

We found 283 lichens species and one subspecific taxon for the Rodnei Mountains in 56 plots (Table 3). We confirmed 102 species that were reported previously, plus 182 taxa that were new records for the region, of which 67 species have never been reported in Romania. Poorly developed specimens that could not be unambiguously assigned to listed species are not reported as new. Considering previous reports and our results, 442 lichen taxa are reported in Rodnei Mountains region, accounting for approx. $35 \%$ of the total lichen flora of Romania. Out of 284 taxa, 13 are listed in the Red List of macrolichens of Romania (Bartok \& Crișan, personal communication), 8 species in the Red List of Ukraine (Didukh, 2009), 65 in the Red List of Hungary (Lökös \& Tóth, 1996), 96 in the Red List of Slovakia (Pisút et al., 2001), and 125 in the Red List of Poland (Cieslinski et al., 2003).

The most species-rich genus is Cladonia, of which we found 43 species in our study area. Even though most species are common, this genus contributed substantially to the lichen species richness of Rodnei Mountains.

Table 2. Red Lists (RL) including the number of lichen species according to IUCN categories: RE - Regionally extinct, CR - Critically Endangered, EN - Endangered, VU - Vulnerable, NT - Near Threatened, LC - Least Concern, DD - Data Deficient, additional category in RL of Hungary: R rare (problematic species including literature data without voucher specimens and new findings with no further information on the distribution), and in RL of Ukraine: R - rare (species known from few locations, with relatively stable populations but low rates), and Total no of sp. - total number of species in the Red List.

\begin{tabular}{|c|c|c|c|c|c|c|c|c|c|}
\hline \multirow{2}{*}{ RL } & \multicolumn{7}{|c|}{ IUCN Categories } & \multirow{2}{*}{$\mathrm{R}$} & \multirow{2}{*}{$\begin{array}{c}\text { Total no of } \\
\text { sp. }\end{array}$} \\
\hline & RE & $\mathrm{CR}$ & $\mathrm{EN}$ & VU & NT & LC & $\mathrm{DD}$ & & \\
\hline Romania & & 20 & 27 & 26 & & & & & 73 \\
\hline Hungary & 30 & 24 & 44 & 45 & & & & 260 & 403 \\
\hline Ukraine & & & 5 & 26 & & & & 21 & 52 \\
\hline Slovakia & 88 & 140 & 48 & 169 & 114 & & 14 & & 573 \\
\hline Poland & 141 & 179 & 201 & 165 & 68 & 22 & 110 & & 886 \\
\hline
\end{tabular}


Table 3. Lichen species recorded in the study area. Abbreviations: New R Mts - new records for the Rodnei Mountains, New RO - new records for Romania; RL RO - Red List of Macrolichens from Romania (Bartok \& Crișan, personal communication), RL UK - Red List of lichens from Ukraine (Didukh, 2009), RL HU - Red List of lichens from Hungary (Lőkös \& Tóth, 1996), RL PL - Red List of lichens from Poland (Cieslinski et al., 2003), RL SL - Red List of lichens from Slovakia (Pisút et al., 2001); Obs - number of plots where the species was observed; categories of red lists: RE - Regionally extinct, CR - Critically Endangered, EN - Endangered, VU - Vulnerable, NT - Near Threatened, LC - Least Concern, DD - Data Deficient, R - rare; poorly developed specimens are marked with *

\begin{tabular}{|c|c|c|c|c|c|c|c|c|c|}
\hline Lichen taxa & $\begin{array}{c}\text { New R } \\
\text { Mts }\end{array}$ & $\begin{array}{l}\mathrm{New} \\
\mathrm{RO}\end{array}$ & $\begin{array}{l}\mathrm{RL} \\
\mathrm{RO}\end{array}$ & $\begin{array}{l}\mathrm{RL} \\
\mathrm{UK} \\
\end{array}$ & $\begin{array}{l}\mathrm{RL} \\
\mathrm{HU} \\
\end{array}$ & $\begin{array}{l}\mathrm{RL} \\
\mathrm{PL}\end{array}$ & $\begin{array}{l}\mathrm{RL} \\
\mathrm{SL}\end{array}$ & Obs & Plot codes \\
\hline Absconditella lignicola Vězda \& Pišút & 1 & 1 & & & & $\mathrm{VU}$ & & 3 & $\mathrm{CC} 1,3,4$ \\
\hline Acrocordia gemmata (Ach.) A. Massal. & & & & & & & & 1 & MC3 \\
\hline Alectoria ochroleuca (Hoffm.) A. Massal. & & & & & & $\mathrm{LC}$ & & 7 & $\mathrm{AC} 1,3,4,5,6 \mathrm{AM} 2,4$ \\
\hline $\begin{array}{l}\text { Amandinea punctata (Hoffm.) Coppins \& } \\
\text { Scheid. }\end{array}$ & & & & & & & & 4 & WM2, 3, 4, 5 \\
\hline Anisomeridium biforme (Borrer) R.C. Harris & 1 & & & & & VU & $\mathrm{RE}$ & 1 & MC5 \\
\hline Anzina carneonivea (Anzi) Scheid. & 1 & & & & & EN & $\mathrm{VU}$ & 1 & PM7 \\
\hline Arthonia caesia (Flot.) Körb. & 1 & 1 & & & & & & 1 & MC3 \\
\hline Arthonia didyma Körb. & 1 & & & & $\mathrm{R}$ & EN & $\mathrm{CR}$ & 2 & MC6, MM7 \\
\hline Arthonia elegans (Ach.) Almq. & 1 & & & & & $\mathrm{RE}$ & & 1 & MC5 \\
\hline Arthonia lapidicola (Taylor) Branth \& Rostr. & & & & & $\mathrm{R}$ & NT & & 2 & MC1, MC6, \\
\hline Arthonia punctiformis Ach. & 1 & & & & $\mathrm{R}$ & EN & $\mathrm{CR}$ & 1 & CM3 \\
\hline Arthonia radiata (Pers.) Ach. & & & & & & & $\mathrm{CR}$ & 6 & $\begin{array}{l}\text { MC5, MC6, MC7, MM4, } \\
\text { MM6, MM7 }\end{array}$ \\
\hline Arthonia vinosa Leight. & & & & & & NT & CR & 1 & MC6 \\
\hline Arthrorhaphis citrinella (Ach.) Poelt & 1 & & & & $\mathrm{VU}$ & VU & & 6 & $\begin{array}{l}\text { AC1, AC3, AC5, AC6, AC7, } \\
\text { PM5 }\end{array}$ \\
\hline Bacidia delicata (Larbal. ex Leight.) Coppins & 1 & 1 & & & & & & 1 & MM3 \\
\hline Bacidia rubella (Hoffm.) A. Massal. & 1 & & & & & VU & VU & 2 & MC3, MM1 \\
\hline Baeomyces placophyllus Ach. & 1 & & & & & $\mathrm{CR}$ & & 4 & PM3, AC6, AM2, CC2 \\
\hline Baeomyces rufus (Huds.) Rebent. & & & & & & & & 27 & $\begin{array}{l}\text { MC1, 2, MC2, MM5, PM3, } \\
7, \text { WM1, 4, 5, AC1, 2, 3, 4, 6, } \\
\text { CC1, 2, 3, 4, 5, 6, 7, CM2, 3, 4, } \\
\text { 5, AM1, 2, } 7\end{array}$ \\
\hline $\begin{array}{l}\text { Bellemerea cinereorufescens (Ach.) Clauzade } \\
\quad \& \mathrm{Cl} \text {. Roux }\end{array}$ & 1 & & & & & $\mathrm{CR}$ & & 1 & MC3 \\
\hline Biatora betulicola Kullh. & 1 & 1 & & & & & & 3 & PM5, AC2, AC3 \\
\hline Biatora chrysantha (Zahlbr.) Printzen & 1 & 1 & & & & & & 2 & PM1, CM6 \\
\hline Biatora efflorescens (Hedl.) Räsänen & 1 & 1 & & & & VU & VU & 1 & CC6 \\
\hline Biatora globulosa (Flörke) Fr. & & & & & $\mathrm{R}$ & & & 2 & MC3, MC4 \\
\hline Brodoa intestiniformis (Vill.) Goward & 1 & & & & & $\mathrm{VU}$ & NT & 6 & $\mathrm{AC} 1,2,4, \mathrm{AM} 1,2,6$ \\
\hline $\begin{array}{l}\text { Bryoria capillaris (Ach.) Brodo \& D. } \\
\text { Hawksw. }\end{array}$ & 1 & & & & & $\mathrm{CR}$ & $\mathrm{CR}$ & 2 & $\mathrm{CC} 1,4$ \\
\hline $\begin{array}{l}\text { Bryoria chalybeiformis (L.) Brodo \& D. } \\
\text { Hawksw. }\end{array}$ & & & & & $\mathrm{VU}$ & $\mathrm{CR}$ & & 1 & AC3 \\
\hline $\begin{array}{l}\text { Bryoria implexa (Hoffm.) Brodo \& D. } \\
\text { Hawksw. }\end{array}$ & & & & & $\mathrm{VU}$ & CR & CR & 5 & AC3, WM3, CC1, 4, 5 \\
\hline Bryoria lanestris (Ach.) Brodo \& D. Hawksw. & 1 & & $\mathrm{VU}$ & & & $\mathrm{RE}$ & $\mathrm{CR}$ & 1 & CC3 \\
\hline Buellia disciformis (Fr.) Mudd & 1 & & & & & $\mathrm{VU}$ & & 3 & MC3, MM3, 5 \\
\hline Buellia erubescens Arnold & & & & & & CR & & 5 & MC2, 3, 4, 7, MM7 \\
\hline $\begin{array}{l}\text { Buellia griseovirens (Turner \& Borrer ex Sm.) } \\
\text { Almb. }\end{array}$ & 1 & 1 & & & & & & 3 & WM5, 7, CM5, \\
\hline Buellia cf. hyperbolica Bagl. & & & & & & & & 1 & PM1 \\
\hline
\end{tabular}


Table 3 (continued)

\begin{tabular}{l} 
Lichen taxa \\
\hline Calicium cf. salicinum ${ }^{*}$ Pers. \\
Candelariella reflexa (Nyl.) Lettau \\
Candelariella vitellina (Ehrh.) Müll. Arg. \\
Catillaria nigroclavata (Nyl.) J. Steiner \\
Cetraria aculeata (Schreb.) Fr. \\
Cetraria ericetorum Opiz \\
Cetraria islandica (L.) Ach.
\end{tabular}

Cetraria muricata (Ach.) Eckfeldt

Cetraria sepincola (Ehrh.) Ach.

Cetrelia olivetorum (Nyl.) W.L. Culb. \& C.F. Culb.

Chaenotheca brachypoda (Ach.) Tibell

Chaenotheca brunneola (Ach.) Müll. Arg.

Chaenotheca chrysocephala (Ach.) Th. Fr. 1

Chaenotheca ferruginea (Turner ex Sm.) Mig. 1

Chaenotheca furfuracea (L.) Tibell $\quad 1$

Chaenotheca gracilenta (Ach.) Mattsson \& 1 Middelb.

Chaenotheca stemonea (Ach.) Müll. Arg. 1

Chaenotheca trichialis (Ach.) Hellb.

Chrysothrix candelaris (L.) J.R. Laundon 1

Cladonia arbuscula (Wallr.) Flot.

Cladonia bellidiflora (Ach.) Schaer.

Cladonia caespiticia (Pers.) Flörke

Cladonia callosa Delise ex Harm.

Cladonia carneola (Fr.) Fr.

Cladonia cenotea (Ach.) Schaer.

Cladonia cervicornis (Ach.) Flot.

Cladonia chlorophaea (Flörke ex Sommerf.) $\quad 1$ Spreng.

Cladonia coccifera (L.) Willd.

EN

Cladonia coniocraea (Flörke) Spreng.

Cladonia cornuta (L.) Hoffm.

VU

Cladonia deformis (L.) Hoffm.

Cladonia digitata (L.) Hoffm.

Cladonia diversa Asperges

$1 \quad 1$

Cladonia fimbriata (L.) Fr.

1

1

1

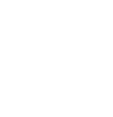

VU

EN

$\begin{array}{llllllll}\text { New R New } & \text { RL } & \text { RL } & \text { RL } & \text { RL } & \text { RL } & \text { Obs } & \text { Plot codes }\end{array}$

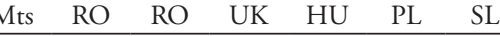

$\begin{array}{clc}\text { EN } & \text { VU } & \text { EN } \\ \text { R } & & \\ & & \\ \text { R } & & \\ \text { CR } & & \\ & \text { NT } & \text { VU } \\ \text { EN } & \text { VU } & \text { VU }\end{array}$

1 MC6

4 MC6, MM4, WM5, 7

3 MM5, WM5, 6

1 WM7

$2 \mathrm{AC} 3,5$

$6 \mathrm{AC} 3,5,6,7, \mathrm{AM} 2,5$

24 PM1, 2, 3, 4, 5, 6, 7, PM2, 3, 4, 5, 6, 7, WM5, AC1, 2, 3, 4, 5, 6, 7, CC3, 4, AM11, 2, 3, 4, 5, 6, 7

$\begin{array}{cllll} & \text { NT } & & 1 & \text { AC3 } \\ \text { R } & \text { EN } & \text { VU } & 1 & \text { PM7 } \\ \text { VU } & \text { EN } & & 2 & \text { MC2,3 }\end{array}$

$\begin{array}{lllll} & \text { EN } & \text { CR } & 1 & \text { MC1 } \\ \text { CR } & \text { EN } & \text { CR } & 2 & \text { CC1, CM7 } \\ \text { VU } & & \text { VU } & 5 & \text { MC1, CC1, 4, 5, 6 } \\ \text { EN } & & & 1 & \text { CC4 } \\ \text { EN } & \text { NT } & \text { NT } & 4 & \text { MC6, MM3, 7, CC1 } \\ & & \text { CR } & 1 & \text { MM3 }\end{array}$
$\begin{array}{llll}\text { EN } & \text { CR } 1 & \text { CC6 }\end{array}$
EN NT CR 3 MM4, CC1,5 CR $2 \mathrm{MC2}, 3$
VU
$12 \mathrm{AC} 1,2,3,4,5,6,7, \mathrm{AM} 2,3$, $4,5,6$
EN
EN
CR EN
VU
$8 \mathrm{AC} 4,7, \mathrm{AM} 1,2,3,4,5,6$
3 AC1, CC6, CM5
1 AC2
5 PM6, WM5, CC2, 3, AM2
11 MC2, MM2, 3, PM1, 2, WM1, 3, 6, CC1, 2, CM5
$8 \mathrm{AC} 1,2,3,4,6,7, \mathrm{AM} 1,3$
34 MC5, 6, 7, MM2, 6, PM1, 2, 3, 4, 5, 6, 7, WM1, 3, 4, 5, 6, 7. $\mathrm{AC} 1,2,3,4,5,6,7, \mathrm{CC} 2,3,5$, 6, 7, CM5, AM1, 3, 4, 7

$16 \mathrm{AC} 1,2,3,4,5,6,7, \mathrm{CC} 3, \mathrm{AM} 1$, 2, 3, 4, 5, 6, 7, PM7

$28 \mathrm{MC1}, 2,3,4,5,6,7, \mathrm{MM}$, 3, 4, 5, 6, 7, PM3, WM1, 3, 4, 6, 7, CC1, 3, 5, 6, 7, CM5, 6, AC5, AM3

VU

13 MC2, 4, 5, 7, MM2, 5, 6, PM1, 3, WM3, CC1, CM5, AM7

4 PM1, CC3, CC5, AM2

$41 \mathrm{MC1}, 2,3,4,5,6,7, \mathrm{MM}$, 3, 4, 6, PM1, 2, 3, 4, 5, 6, WM1, $2,3,6, \mathrm{AC} 1,2,3,4,5,6, \mathrm{CC} 1$ 2, 3, 4, 5, 6, 7, CM1, 2, 5, AM1, $2,3,7$

3 AC3, AC4, AM1

15 MC4, 5, 7, MM2, 5, 6, WM1, 2, 4, 5, 6, 7, CC3, 7, СM5, 
Table 3 (continued)

\begin{tabular}{|c|c|c|c|c|c|c|c|c|c|}
\hline Lichen taxa & $\begin{array}{l}\text { New R } \\
\text { Mts }\end{array}$ & $\begin{array}{l}\text { New } \\
\text { RO }\end{array}$ & $\begin{array}{l}\mathrm{RL} \\
\mathrm{RO}\end{array}$ & $\begin{array}{l}\mathrm{RL} \\
\mathrm{UK}\end{array}$ & $\begin{array}{l}\text { RL } \\
\mathrm{HU}\end{array}$ & $\begin{array}{l}\text { RL } \\
\mathrm{PL}\end{array}$ & $\begin{array}{l}\mathrm{RL} \\
\mathrm{SL}\end{array}$ & Obs & Plot codes \\
\hline Cladonia floerkeana (Fr.) Flörke & & & & & $\mathrm{EN}$ & & & 13 & $\begin{array}{l}\mathrm{MM} 2,3, \mathrm{AC} 3,4,5,6,7, \mathrm{CC} 3 \\
6, \mathrm{AM} 2,3,4,7\end{array}$ \\
\hline Cladonia furcata (Huds.) Schrad. & & & & & & & & 17 & $\begin{array}{l}\text { MC7, MM2, 5, 6, WM1, 2, 3, 5, } \\
\text { 6, 7, CC1, 3, 4, 5, 6, CM5, AM7 }\end{array}$ \\
\hline Cladonia gracilis (L.) Willd. & & & & & VU & & & 9 & $\begin{array}{l}\text { MC5, PM4, 5, 6, AC2, 5, CC3, } \\
5,6\end{array}$ \\
\hline Cladonia humilis (With.) J.R. Laundon & 1 & 1 & & & & & & 6 & MC6, WM2, 3, 5, 7, AM6 \\
\hline Cladonia incrassata Flörke & 1 & & EN & & & $\mathrm{EN}$ & $\mathrm{RE}$ & 4 & $\mathrm{AC} 1,7, \mathrm{CM} 1$ \\
\hline Cladonia luteoalba A. Wilson \& Wheldon & 1 & 1 & & & & & & 1 & AC5 \\
\hline Cladonia macilenta Hoffm. & & & & & & & & 21 & $\begin{array}{l}\text { MC7, MM2, 3, 5, 6, PM2, 4, 6, } \\
\text { WM1, 5, 6, 7, AC4, 6, CC2, 5, } \\
\text { 6, AM1, 3, 5, 6 }\end{array}$ \\
\hline Cladonia macroceras (Delise) Ahti & 1 & 1 & & & & $\mathrm{VU}$ & & 21 & $\begin{array}{l}\text { PM1, 2, 3, 4, 5, 6, 7, AC1, 2, 3, } \\
5,6,7, \text { AM1 } 2,3,5,6,7\end{array}$ \\
\hline Cladonia macrophylla (Schaer.) Stenh. & & & VU & & & CR & EN & 1 & AC7 \\
\hline Cladonia magyarica Vain. ex Gyeln. & 1 & 1 & & & & & CR & 1 & PM4 \\
\hline Cladonia maxima (Asahina) Ahti & 1 & 1 & & & & & & 11 & $\begin{array}{l}\text { PM1, 2, 3, AC1, 6, 7, AM1, 2, } \\
3,5,6\end{array}$ \\
\hline Cladonia metacorallifera Asahina & 1 & 1 & & & & & & 2 & $\mathrm{AC} 3,4$ \\
\hline Cladonia ochrochlora Flörke & & & & & $\mathrm{R}$ & & & 19 & $\begin{array}{l}\text { MC1, 2, 4, 5, 6, 7, MM2, 3, } \\
\text { 4, 6, PM6, WM1, 2, 3, 5, 6, 7, } \\
\text { CC1, CM5 }\end{array}$ \\
\hline Cladonia parasitica (Hoffm.) Hoffm. & 1 & & & & & $\mathrm{EN}$ & NT & 36 & $\begin{array}{l}\mathrm{MC} 1,2,3,5,6,7, \mathrm{MM} 1,2,3, \\
\text { 6, 7, PM1, 2, 3, 6, 7, WM1, 2, } \\
3,4,6,7, \mathrm{AC} 1,4, \mathrm{CC} 1,2,3,6, \\
\text { 7, CM1, 2, 4, 6, AM6 }\end{array}$ \\
\hline Cladonia phyllophora Ehrh. ex Hoffm. & 1 & & & & $\mathrm{R}$ & & & 6 & $\begin{array}{l}\text { AC1, AC2, AC3, AM1, AM4, } \\
\text { AM6 }\end{array}$ \\
\hline Cladonia pleurota (Flörke) Schaer. & 1 & & & & $\mathrm{EN}$ & & & 10 & $\begin{array}{l}\mathrm{PM} 1,3,4, \mathrm{AC} 2,3,4,6,7, \\
\mathrm{PP} 6,7\end{array}$ \\
\hline Cladonia pocillum (Ach.) O.J. Rich. & & & & & & & & 4 & $\mathrm{AC} 2, \mathrm{AM} 1,3,5$ \\
\hline Cladonia polydactyla (Flörke) Spreng. & 1 & & & & $\mathrm{EN}$ & & & 31 & $\begin{array}{l}\text { MC1, 2, 4, 5, MM5, 6, PM1, } 2 \\
3,4,5,6,7, \text { WM1 } 2,6, \text { AC } 3 \\
4,5,6,7, \text { CC1, 3, 5, 6, CM5, } \\
\text { AM2, 3, 4, 6, } 7\end{array}$ \\
\hline Cladonia pyxidata (L.) Hoffm. & & & & & & & & 22 & $\begin{array}{l}\text { MC6, MM3, PM1, 2, 3, 4, 5, } \\
\text { WM1, 6, AC1, 2, 3, 4, 5, 6, } \\
\text { 7,CM5, AM2, 3, 4 }\end{array}$ \\
\hline Cladonia ramulosa (With.) J.R. Laundon & 1 & & & & & & & 4 & PM1, WM1, AC2, CM5, \\
\hline $\begin{array}{l}\text { Cladonia rangiferina (L.) Weber ex F.H. } \\
\text { Wigg. }\end{array}$ & & & & & VU & & & 18 & $\begin{array}{l}\mathrm{PM} 1,2,4,5,6, \mathrm{AC} 1,2,3,4,5 \\
6,7, \mathrm{CC} 3, \mathrm{AM} 1,3,4,5,6\end{array}$ \\
\hline Cladonia rangiformis Hoffm. & & & & & & & & 3 & CC5, AM5, 6 \\
\hline Cladonia squamosa (Scop.) Hoffm. & & & & & & & & 32 & $\begin{array}{l}\text { MC5, MM7, PM1, 2, 3, 4, 5, 6, } \\
\text { 7, WM1, 3, AC1, 2, 3, 4, 5, 6, 7, } \\
\text { CC1, 2, 5, 6, AM1, 2, 3, WM1, } \\
\text { CC } 3,4, \text { CM2, 3, AM7 }\end{array}$ \\
\hline Cladonia subcervicornis (Vain.) Kernst. & 1 & 1 & & & & CR & & 18 & $\begin{array}{l}\text { MC2, 5, 6, MM2, 6, PM1, } \\
\text { WM1, 5, AC1, 2, 4, 5, 6, 7, } \\
\text { CC2, 4, AM3, 7 }\end{array}$ \\
\hline Cladonia subulata (L.) Weber ex F.H. Wigg. & 1 & & & & & & & 15 & $\begin{array}{l}\text { MM2, 4, 6, PM1, WM1, 2, 4, 5, } \\
\text { 6, 7, AC2, 3, 5, CC3, AM2 }\end{array}$ \\
\hline Cladonia sulphurina (Michx.) Fr. & 1 & & $\mathrm{CR}$ & & & NT & EN & 7 & $\begin{array}{l}\text { PM4, PM5, AC3, AC5, AC7, } \\
\text { AM2, AM5 }\end{array}$ \\
\hline Cladonia symphycarpia (Flörke) Fr. & 1 & & & & & & & 1 & AM5 \\
\hline
\end{tabular}


Table 3 (continued)

\begin{tabular}{|c|c|c|c|c|c|c|c|c|c|}
\hline Lichen taxa & $\begin{array}{l}\text { New R } \\
\text { Mts }\end{array}$ & $\begin{array}{l}\text { New } \\
\text { RO }\end{array}$ & $\begin{array}{l}\mathrm{RL} \\
\mathrm{RO}\end{array}$ & $\begin{array}{l}\text { RL } \\
\text { UK }\end{array}$ & $\begin{array}{l}\mathrm{RL} \\
\mathrm{HU}\end{array}$ & $\begin{array}{l}\mathrm{RL} \\
\mathrm{PL}\end{array}$ & $\begin{array}{l}\mathrm{RL} \\
\mathrm{SL}\end{array}$ & Obs & Plot codes \\
\hline Cladonia uncialis (L.) Weber exF.H. Wigg. & 1 & & & & VU & & & 7 & $\begin{array}{l}\text { AC3, AC4, AM1, AM2, AM4, } \\
\text { AM5, AM6 }\end{array}$ \\
\hline Collema flaccidum (Ach.) Ach. & & & & & & $\mathrm{EN}$ & $\mathrm{EN}$ & 3 & $\mathrm{MC} 3,4,6$ \\
\hline $\begin{array}{l}\text { Cornicularia normoerica (Gunnerus) Du } \\
\quad \text { Rietz }\end{array}$ & & & & & & $\mathrm{VU}$ & & 7 & PM5, AC1, 3, 4, 5, 7, AM1 \\
\hline Dibaeis baeomyces (L. f.) Rambold \& Hertel & & & & & & NT & & 1 & AC3 \\
\hline Dimerella pineti (Ach.) Vězda & 1 & & & & & & & 6 & MM2, 4, CC6, CM4, 6, 7 \\
\hline Ephebe lanata (L.) Vain. & & & & & & $\mathrm{EN}$ & & 1 & AC4 \\
\hline Evernia divaricata (L.) Ach. & & & & & & CR & CR & 4 & MC4, MM3, 5, CC4 \\
\hline Evernia prunastri (L.) Ach. & & & & & & NT & $\mathrm{EN}$ & 11 & $\begin{array}{l}\text { MC2, 3, 4, 5, 6, 7, MM1, } \\
\text { WM2, 3, CC1, 6 }\end{array}$ \\
\hline $\begin{array}{l}\text { Fellhaneropsis vezdae (Coppins \& P. James) } \\
\text { Sérus. \& Coppins }\end{array}$ & 1 & 1 & & & & $\mathrm{LC}$ & & 1 & MM4 \\
\hline $\begin{array}{l}\text { Flavocetraria cucullata (Bellardi) Kärnefelt } \\
\text { \& A. Thell }\end{array}$ & & & & & & $\mathrm{VU}$ & & 2 & $\mathrm{AM} 2,4$ \\
\hline Fuscidea arboricola Coppins \& Tønsberg & 1 & 1 & & & & & & 8 & $\mathrm{MM} 2,3, \mathrm{CC} 2,5,6,7, \mathrm{CM} 6$ \\
\hline Fuscidea pusilla Tønsberg & 1 & 1 & & & & & & 7 & $\begin{array}{l}\text { MC6, MM2, WM5, CC5, 7, } \\
\text { CM3, } 4\end{array}$ \\
\hline Graphis pulverulenta (Pers.) Ach. & 1 & & & & & & & 5 & $\mathrm{MC} 1,3,7, \mathrm{MM} 2,7$ \\
\hline Graphis scripta (L.) Ach. & & & & & & NT & $\mathrm{EN}$ & 5 & $\mathrm{MC} 1,3,4,5,6$ \\
\hline Heterodermia speciosa (Wulfen) Trevis. & 1 & & & $\mathrm{R}$ & & CR & CR & 1 & MC5 \\
\hline $\begin{array}{l}\text { Hypocenomyce scalaris (Ach. ex Lilj.) M. } \\
\text { Choisy }\end{array}$ & & & & & & & & 1 & WM7 \\
\hline Hypogymnia farinacea Zopf & 1 & & & & & $\mathrm{VU}$ & VU & 4 & WM3, 7, CC6, CM5, \\
\hline Hypogymnia physodes (L.) Nyl. & & & & & & & & 39 & $\begin{array}{l}\mathrm{MC} 1,2,3,4,5,6,7, \mathrm{MM} 1,2, \\
3,4,5,6,7, \mathrm{PM} 2,3,4,6, \mathrm{WM} 1, \\
2,3,4,5,6,7, \mathrm{AC} 3, \mathrm{CC} 1,2,3, \\
4,5,6,7, \mathrm{CM} 1,2,3,4,5,6\end{array}$ \\
\hline Hypogymnia tubulosa (Schaer.) Hav. & & & & & VU & NT & NT & 14 & $\begin{array}{l}\text { MC3, 5, 7, MM3, PM4, WM1, } \\
2,3,4,6,7, \mathrm{CC} 3,4, \mathrm{CM} 6\end{array}$ \\
\hline Hypogymnia vittata (Ach.) Parrique & & & & & & CR & VU & 1 & AC6 \\
\hline Icmadophila ericetorum (L.) Zahlbr. & 1 & & & & & EN & CR & 2 & AC3, CC3 \\
\hline Imshaugia aleurites (Ach.) S.L.F. Mey. & & & & & & & VU & 1 & WM4 \\
\hline Japewia subaurifera Muhr \& Tønsberg & 1 & 1 & & & & & & 2 & MM1, CC2 \\
\hline Lecanactis abietina (Ehrh. ex Ach.) Körb. & 1 & & & & & & CR & 1 & $\mathrm{CC} 1$ \\
\hline Lecanora albella (Pers.) Ach. & & & & & & EN & CR & 1 & MC4 \\
\hline Lecanora albellula (Nyl.) Th. & 1 & 1 & & & & & & 2 & WM2, 4 \\
\hline Lecanora argentata (Ach.) Malme & 1 & & & & & & & 4 & $\mathrm{MC} 3,4,5,6$ \\
\hline Lecanora carpinea (L.) Vain. & 1 & & & & & & & 8 & MC2, 3, 4, 5, 6, 7, MM5, WM2 \\
\hline Lecanora cf. hybocarpa (Tuck.) Brodo & & & & & & & & 1 & MC5 \\
\hline Lecanora chlarotera $\mathrm{Nyl}$. & 1 & & & & & & & 17 & $\begin{array}{l}\text { MC1, 2, 6, 7, MM2, 3, 4, 5, 6, } \\
\text { 7, WM2, 3, 4, 5, 6, 7, CC4 }\end{array}$ \\
\hline Lecanora cinereofusca $\mathrm{H}$. Magn. & 1 & & & & & & CR & 3 & $\mathrm{MC} 3,4,5$ \\
\hline Lecanora circumborealis Brodo \& Vitik. & 1 & 1 & & & & & & 4 & MC6, WM3, 4, 5 \\
\hline Lecanora compallens Herk \& Aptroot & 1 & 1 & & & & & & 2 & AC3, 5 \\
\hline Lecanora conizaeoides Nyl. ex Cromb. & 1 & & & & & & & 10 & $\begin{array}{l}\text { MM3, WM1, CC3, 4, 5, 6, } \\
\text { CM3, 4, 5, 6 }\end{array}$ \\
\hline Lecanora expallens Ach. & 1 & & & & & & & 4 & $\mathrm{MC} 1,4, \mathrm{CC} 1,5$ \\
\hline Lecanora glabrata (Ach.) Malme & 1 & & & & & & & 1 & WM5 \\
\hline Lecanora intumescens (Rebent.) Rabenh. & & & & & & $\mathrm{EN}$ & $\mathrm{EN}$ & 6 & $\mathrm{MC} 1,2,4,6,7, \mathrm{CC} 2$ \\
\hline
\end{tabular}


Table 3 (continued)

\begin{tabular}{|c|c|c|c|c|c|c|c|c|c|}
\hline Lichen taxa & $\begin{array}{l}\text { New R } \\
\text { Mts }\end{array}$ & $\begin{array}{l}\text { New } \\
\text { RO }\end{array}$ & $\begin{array}{l}\mathrm{RL} \\
\mathrm{RO}\end{array}$ & $\begin{array}{l}\text { RL } \\
\text { UK }\end{array}$ & $\begin{array}{l}\mathrm{RL} \\
\mathrm{HU}\end{array}$ & $\begin{array}{l}\text { RL } \\
\text { PL }\end{array}$ & $\begin{array}{l}\text { RL } \\
\text { SL }\end{array}$ & Obs & Plot codes \\
\hline Lecanora pulicaris (Pers.) Ach. & 1 & & & & & & & 23 & $\begin{array}{l}\text { MC1, 2, 5, MC6, 7, PM5, 6, 7, } \\
\text { WM1, 2, 3, 4, 5, 6, 7, CC2, 3, 6, } \\
\text { 7, CM3 } 4,5,6\end{array}$ \\
\hline Lecanora saligna (Schrad.) Zahlbr. & 1 & & & & & & & 1 & WM3 \\
\hline Lecanora strobilina (Spreng.) Kieff. & 1 & 1 & & & & & & 7 & $\begin{array}{l}\text { MC6, MM2, 4, 5, PM5, WM2, } \\
7\end{array}$ \\
\hline Lecanora subintricata (Nyl.) Th. Fr. & 1 & & & & & & & 4 & PM6, WM2, 4, 7 \\
\hline Lecanora subrugosa $\mathrm{Nyl}$. & 1 & & & & & $\mathrm{LC}$ & & 1 & MM4 \\
\hline Lecanora symmicta (Ach.) Ach. & 1 & & & & & & & 5 & PM2, 5, 6, WM2, 4 \\
\hline Lecanora umbrina (Ehrh.) Röhl & 1 & & & & & & & & WM7 \\
\hline Lecanora varia (Hoffm.) Ach. & 1 & & & & $\mathrm{R}$ & & VU & 4 & WM2, 3, 4, 5 \\
\hline Lecidea berengeriana (A. Massal.) $\mathrm{Nyl}$. & 1 & & & & & & & 1 & AC5 \\
\hline Lecidea leprarioides Tønsberg & 1 & 1 & & & & & & 1 & $\mathrm{CC} 1$ \\
\hline Lecidea nylanderi (Anzi) Th. Fr. & 1 & 1 & & & & & & 24 & $\begin{array}{l}\mathrm{MC} 1,2,3,4,5,7, \mathrm{MM} 1,2,4 \\
5,7, \mathrm{PM} 1, \mathrm{WM} 5, \mathrm{CC} 1,2,3,4, \\
5,6,7, \mathrm{CM} 1,2,3,4\end{array}$ \\
\hline Lecidella cf. albida Hafellner & & & & & & & & 1 & MC3 \\
\hline Lecidella elaeochroma (Ach.) M. Choisy & & & & & & & & 9 & $\mathrm{MC} 1,2,3,4,5,6,7, \mathrm{MM} 1,4$ \\
\hline $\begin{array}{l}\text { Lecidella elaeochroma f. soralifera (Erichsen) } \\
\text { D. Hawksw. }\end{array}$ & 1 & 1 & & & & & & 1 & $\mathrm{CC} 2$ \\
\hline Lecidella subviridis Tønsberg & 1 & 1 & & & & & & 10 & $\begin{array}{l}\text { MC5, 6, 7, MM1, 2, 7, CC2, } \\
3,6,7\end{array}$ \\
\hline $\begin{array}{l}\text { Lecidoma demissum (Rutstr.) Gotth. Schneid. } \\
\quad \& \text { Hertel }\end{array}$ & & & & & & $\mathrm{EN}$ & & 5 & $\mathrm{AC} 2,4,7, \mathrm{AM} 1,3$ \\
\hline Lepraria eburnea J.R. Laundon & 1 & 1 & & & & & & 11 & $\begin{array}{l}\text { MC1, 6, 7, MM1, 2, 4, 5, 7, } \\
\text { CM1, 4, 7 }\end{array}$ \\
\hline Lepraria ecorticata (J.R. Laundon) Kukwa & 1 & 1 & & & & & & 2 & PM4, CC1 \\
\hline Lepraria elobata Tønsberg & 1 & 1 & & & & & & 24 & $\begin{array}{l}\text { MC2, 3, 4, 5, 6, MM6, 7, PM1, } \\
\text { 2, 3, WM6, AC2, 4, CC2, 3, 4, } \\
\text { 5, 6, 7, CM2, 3, 4, 5, } 6\end{array}$ \\
\hline Lepraria incana (L.) Ach. & 1 & & & & & & & 1 & MC3 \\
\hline Lepraria jackii Tønsberg & 1 & 1 & & & & & & 17 & $\begin{array}{l}\text { MC1, 2, MM1, 3, 7, PM5, } \\
\text { WM5, AC5, CC2, 3, 4, 5, 6, } \\
\text { CM1, 3, 4, } 7\end{array}$ \\
\hline Lepraria lobificans $\mathrm{Nyl}$. & 1 & & & & $\mathrm{R}$ & & & 32 & $\begin{array}{l}\mathrm{MC} 1,2,3,4,5,6,7, \mathrm{MM} 1,2 \\
3,4,5,6,7, \mathrm{PM} 2,3,7, \mathrm{WM} 1, \\
4, \mathrm{AC} 2, \mathrm{CC} 1,2,3,4,5,6,7, \\
\mathrm{CM} 2,3,4,6,7\end{array}$ \\
\hline Lepraria membranacea (Dicks.) Vain. & 1 & & & & & & & 2 & MM2, CC1 \\
\hline Lepraria neglecta Vain. & 1 & 1 & & & & & & 1 & AC4 \\
\hline Lepraria nivalis J.R. Laundon & 1 & 1 & & & & & & 11 & $\begin{array}{l}\text { PM2, 5, WM2, AC2, 3, 4, 5, 6, } \\
\text { 7, CC2, AM7 }\end{array}$ \\
\hline Lepraria rigidula (B. de Lesd.) Diederich & 1 & 1 & & & & & & 7 & $\begin{array}{l}\text { MC2, 6, MM1, WM2, CC1, } \\
\text { 5, CM3 }\end{array}$ \\
\hline Lepraria toensbergiana Slav.-Bay. \& Kukwa & 1 & 1 & & & & & & 8 & $\begin{array}{l}\text { MC7, MM3, PM3, CC1, 3, 7, } \\
\text { CM3, 4, }\end{array}$ \\
\hline Lepraria umbricola Tønsberg & 1 & 1 & & & & & & 3 & MM1, CM2, 7 \\
\hline Lepraria vouauxii (Hue) RC. Harris & 1 & 1 & & & $\mathrm{R}$ & & & 3 & MM2, CM1, 7 \\
\hline Leprocaulon microscopicum (Vill.) Gams & 1 & & & & & & & 2 & MC5, CC4 \\
\hline Leptogium lichenoides (L.) Zahlbr. & & & & & & $\mathrm{LC}$ & & 5 & MC3, 6, MM5, 6, 7 \\
\hline $\begin{array}{l}\text { Lichenomphalia hudsoniana (H.S. Jenn.) } \\
\text { Redhead et al. }\end{array}$ & 1 & & & $\mathrm{R}$ & & NT & VU & 5 & PM5, AC3, CC6, CM6, AM2 \\
\hline $\begin{array}{l}\text { Lichenomphalia umbellifera (L.) Redhead } \\
\text { et al. }\end{array}$ & 1 & 1 & & & & NT & & 3 & $\mathrm{CM} 3,6$ \\
\hline
\end{tabular}


Table 3 (continued)

\begin{tabular}{|c|c|c|c|c|c|c|c|c|c|}
\hline Lichen taxa & $\begin{array}{l}\text { New R } \\
\text { Mts }\end{array}$ & $\begin{array}{l}\text { New } \\
\text { RO }\end{array}$ & $\begin{array}{l}\mathrm{RL} \\
\mathrm{RO}\end{array}$ & $\begin{array}{l}\text { RL } \\
\text { UK }\end{array}$ & $\begin{array}{l}\text { RL } \\
\mathrm{HU}\end{array}$ & $\begin{array}{l}\mathrm{RL} \\
\mathrm{PL}\end{array}$ & $\begin{array}{l}\mathrm{RL} \\
\mathrm{SL}\end{array}$ & Obs & Plot codes \\
\hline $\begin{array}{l}\text { Lichenomphalia velutina (Quél.) Redhead } \\
\text { et al. }\end{array}$ & 1 & 1 & & & & & & 1 & $\mathrm{MC1}$ \\
\hline Lobaria pulmonaria (L.) Hoffm. & & & VU & VU & $\mathrm{RE}$ & $\mathrm{EN}$ & CR & 2 & MC3, MM1 \\
\hline Loxospora cismonica (Beltr.) Hafellner & 1 & & & & & $\mathrm{CR}$ & $\mathrm{RE}$ & 2 & $\mathrm{MC1}, 5$ \\
\hline Loxospora elatina (Ach.) A. Massal. & 1 & & & & & $\mathrm{EN}$ & NT & 16 & $\begin{array}{l}\mathrm{MC} 1,2,3,4,5,6,7, \mathrm{MM} 1,2 \\
\mathrm{CC} 1,4,5,6, \mathrm{CM} 2,4,5\end{array}$ \\
\hline Megalospora tuberculosa (Fée) Sipman & 1 & 1 & & & & & & 1 & MC7 \\
\hline Melanelia hepatizon (Ach.) A. Thell & & & & & & & NT & 4 & PM6, AC4, 6, AM5 \\
\hline Melanelia stygia (L.) Essl. & 1 & & & & & & NT & 3 & PM2, AC3, 5 \\
\hline $\begin{array}{l}\text { Melanelixia fuliginosa subsp. glabratula } \\
\text { (Lamy) J.R. Laundon }\end{array}$ & & & & & & & & 23 & $\begin{array}{l}\mathrm{MC} 1,2,3,4,5,7, \mathrm{MM} 1,2,3, \\
\text { 4, 5, 7, WM1,2, 4, 5, 6, 7, CC4, } \\
\text { 7, CM6, } 7\end{array}$ \\
\hline $\begin{array}{l}\text { Melanelixia subargentifera (Nyl.) O. Blanco } \\
\text { et al. }\end{array}$ & 1 & & & & & VU & $\mathrm{VU}$ & 2 & WM2, 3 \\
\hline $\begin{array}{l}\text { Melanelixia subaurifera (Nyl.) O. Blanco } \\
\text { et al. }\end{array}$ & 1 & & VU & & & & NT & 11 & $\begin{array}{l}\mathrm{MC} 2,3,4,5, \mathrm{MM} 2,5, \mathrm{WM} 2 \\
3,5,6,7\end{array}$ \\
\hline $\begin{array}{l}\text { Melanohalea elegantula (Zahlbr.) O. Blanco } \\
\text { et al. }\end{array}$ & 1 & 1 & & $\mathrm{R}$ & & VU & & 2 & MC6, MM1 \\
\hline $\begin{array}{l}\text { Melanohalea exasperatula (Nyl.) O. Blanco } \\
\text { et al. }\end{array}$ & 1 & & & & & & & 6 & MC6, WM2, 3, 4, 5, 7 \\
\hline Melaspilea ochrothalamia $\mathrm{Nyl}$. & 1 & 1 & & & & & & 2 & PM1, PM5 \\
\hline Melaspilea proximella (Nyl.) Nyl. & 1 & & & & & & & 1 & PM4 \\
\hline Menegazzia terebrata (Hoffm.) A. Massal. & 1 & & & & $\mathrm{CR}$ & CR & CR & 1 & MC4 \\
\hline $\begin{array}{l}\text { Micarea elachista (Körb.) Coppins \& R. } \\
\text { Sant. }\end{array}$ & 1 & 1 & & & & $\mathrm{EN}$ & & 2 & WM3 \\
\hline Micarea farinosa Coppins \& Aptroot & 1 & 1 & & & & & & 1 & CM2 \\
\hline Micarea lignaria (Ach.) Hedl. & & & & & $\mathrm{RE}$ & & & 5 & $\mathrm{PM} 2, \mathrm{AC} 3,5,6, \mathrm{AM} 1$ \\
\hline Micarea melaena (Nyl.) Hedl. & 1 & & & & $\mathrm{R}$ & NT & & 1 & CM4 \\
\hline Micarea micrococca (Körb.) Gams & 1 & 1 & & & & & & 1 & MM4, \\
\hline Micarea misella (Nyl.) Hedl. & 1 & & & & $\mathrm{R}$ & & & 2 & MC2, MM5 \\
\hline Micarea prasina Fr. & 1 & & & & & & & 23 & $\begin{array}{l}\mathrm{MC} 1,4,5,7, \mathrm{MM} 1,3,4,5, \\
6,7, \mathrm{PM} 7, \mathrm{CC} 1,2,3,4,6,7 \\
\mathrm{CM} 2,3,4,5,6,7\end{array}$ \\
\hline Micarea turfosa (A. Massal.) Du Rietz & 1 & 1 & & & & DD & & 1 & $\mathrm{AC} 2$ \\
\hline $\begin{array}{l}\text { Micarea viridileprosa Coppins \& Van den } \\
\text { Boom }\end{array}$ & 1 & 1 & & & & & & 1 & $\mathrm{MC1}$ \\
\hline Micarea xanthonica Coppins \& Tønsberg & 1 & 1 & & & & & & 1 & MM5 \\
\hline Mycobilimbia epixanthoides (Stirt.) Zahlbr. & 1 & 1 & & & & & & 10 & $\begin{array}{l}\text { MC5, 7, MM4, 5, 6, 7, AC2, } \\
\text { CC6, 7, CM7 }\end{array}$ \\
\hline Mycoblastus fucatus (Stirt.) Zahlbr. & 1 & 1 & & & & & & 6 & $\mathrm{MC} 2,4,5,7, \mathrm{MM} 3, \mathrm{CC} 5$ \\
\hline Mycoblastus sanguinarius (L.) Norman & 1 & & & & & VU & CR & 1 & MC2 \\
\hline Nephroma parile (Ach.) Ach. & 1 & & & $\mathrm{VU}$ & $\mathrm{CR}$ & CR & CR & 1 & $\mathrm{CC} 2$ \\
\hline Ochrolechia androgyna (Hoffm.) Arnold & 1 & & & & & VU & & 3 & MC6, CC2,AM4 \\
\hline Opegrapha niveoatra (Borrer) J.R. Laundon & 1 & & & & & & & 1 & MM2 \\
\hline Opegrapha varia Pers. & & & & & & NT & VU & 4 & $\mathrm{MC} 1,3,5,6$ \\
\hline Opegrapha viridis Pers. & & & & & $\mathrm{R}$ & VU & $\mathrm{VU}$ & 3 & $\mathrm{MC1}, 3,4$ \\
\hline Opegrapha vulgata (Ach.) Ach. & & & & & & VU & $\mathrm{VU}$ & 2 & $\mathrm{MC} 1,5$ \\
\hline Parmelia omphalodes (L.) Ach. & 1 & & & & & $\mathrm{EN}$ & NT & 4 & PM2, AC3, CC2, AM1, \\
\hline Parmelia saxatilis (L.) Ach. & & & & & & & NT & 12 & $\begin{array}{l}\text { PM6, WM2, 4, AC3, 4, 5, CC2, } \\
\text { 4, AM2, 4, 5, } 6\end{array}$ \\
\hline Parmelia submontana Nádv. & 1 & & & & & VU & CR & 6 & $\mathrm{MC} 2,3,6, \mathrm{WM} 4,5,7$ \\
\hline
\end{tabular}


Table 3 (continued)

\begin{tabular}{|c|c|c|c|c|c|c|c|c|c|}
\hline Lichen taxa & $\begin{array}{l}\text { New R } \\
\text { Mts }\end{array}$ & $\begin{array}{l}\text { New } \\
\text { RO }\end{array}$ & $\begin{array}{l}\mathrm{RL} \\
\mathrm{RO}\end{array}$ & $\begin{array}{l}\mathrm{RL} \\
\mathrm{UK}\end{array}$ & $\begin{array}{l}\mathrm{RL} \\
\mathrm{HU}\end{array}$ & $\begin{array}{l}\text { RL } \\
\text { PL }\end{array}$ & $\begin{array}{l}\text { RL } \\
\text { SL }\end{array}$ & Obs & Plot codes \\
\hline Parmelia sulcata Taylor & & & & & & & & 10 & $\begin{array}{l}\text { MC2, 4, 6, PM6, WM2, 3, 4, 6, } \\
\text { 7, CC5 }\end{array}$ \\
\hline Parmelina quercina (Willd.) Hale & 1 & & & & & $\mathrm{CR}$ & CR & 2 & WM2, 3 \\
\hline Parmeliopsis ambigua (Wulfen) Nyl. & & & & & & & & 18 & $\begin{array}{l}\text { MC2, PM1, 2, 3, 4, 6, 7, WM1, } \\
3,4,6,7, \mathrm{CC} 1,2,3,6,7, \mathrm{CM} 5\end{array}$ \\
\hline Parmeliopsis hyperopta (Ach.) Vain. & & & & & & $\mathrm{VU}$ & VU & 6 & MC4, PM1, 4, 6, 7, CC4 \\
\hline Peltigera canina (L.) Willd. & & & & & & $\mathrm{VU}$ & & 3 & WM3, 5, 6 \\
\hline Peltigera degenii Gyeln. & 1 & & & & EN & $\mathrm{VU}$ & $\mathrm{VU}$ & 3 & MC3, 5, MM5 \\
\hline Peltigera horizontalis (Huds.) Baumg. & & & & & & $\mathrm{EN}$ & & 6 & MC2, 3, 5, MM5, WM3, 4, \\
\hline Peltigera hymenina (Ach.) Delise & 1 & 1 & & & $\mathrm{EN}$ & DD & & 10 & $\begin{array}{l}\text { MC4, 5, 6, MM1, 3, 4, WM2, 3, } \\
\text { CC6, CM2 }\end{array}$ \\
\hline Peltigera lepidophora (Nyl.) Bitter & 1 & & $\mathrm{CR}$ & & & $\mathrm{EN}$ & CR & 1 & MM2 \\
\hline Peltigera membranacea (Ach.) Nyl. & 1 & & & & & DD & & 1 & WM5 \\
\hline Peltigera polydactylon (Neck.) Hoffm. & 1 & & & & EN & DD & & 4 & MC1, MM2, 4, WM5, \\
\hline $\begin{array}{l}\text { Peltigera praetextata (Flörke ex Sommerf.) } \\
\text { Zopf }\end{array}$ & 1 & & & & & $\mathrm{VU}$ & & 8 & $\mathrm{MC1}, 2,3,4, \mathrm{MM} 4,5,6,7$ \\
\hline Peltigera rufescens (Weiss) Humb. & 1 & & & & & & & 3 & WM3, 5,7 \\
\hline Pertusaria amara (Ach.) Nyl. & & & & & & & NT & 2 & $\mathrm{MC} 2,5$ \\
\hline Pertusaria coccodes (Ach.) Nyl. & 1 & 1 & & & $\mathrm{R}$ & NT & $\mathrm{VU}$ & 1 & MM4 \\
\hline Pertusaria leioplaca DC. & & & & & $\mathrm{R}$ & NT & CR & 4 & $\mathrm{MC} 1,2,5,6$, \\
\hline Pertusaria oculata (Dicks.) Th. Fr. & 1 & & & & & & & 1 & AC5 \\
\hline Pertusaria pertusa (L.) Tuck. & & & & & & $\mathrm{VU}$ & NT & 1 & MC3 \\
\hline Pertusaria pupillaris (Nyl.) Th. Fr. & 1 & 1 & & & & NT & & 1 & MC6 \\
\hline Phaeophyscia endophoenicea (Harm.) Moberg & 1 & 1 & & & $\mathrm{R}$ & $\mathrm{EN}$ & CR & 1 & MC6 \\
\hline Phaeophyscia orbicularis (Neck.) Moberg & 1 & & & & & & & 3 & MM4, WM3, 4 \\
\hline Phlyctis argena (Ach.) Flot. & 1 & & & & & & & 6 & $\mathrm{MC} 2,3,4,6, \mathrm{MM} 1,6$ \\
\hline Physcia adscendens (Fr.) H. Olivier & 1 & & & & & & & 6 & MM4, WM2, 3, 4, 6, 7, \\
\hline Physcia aipolia (Ehrh. ex Humb.) Fürnr. & 1 & & & & & NT & & 3 & WM2, 5,7 \\
\hline Physcia dubia (Hoffm.) Lettau & 1 & & & & $\mathrm{R}$ & & & 1 & WM2 \\
\hline Physcia leptalea (Ach.) DC. & 1 & 1 & & & & & & 1 & MM5 \\
\hline Physcia stellaris (L.) Nyl. & & & & & & & & 5 & MC7, WM2, 3, 4, 6 \\
\hline Physcia tenella (Scop.) DC. & 1 & & & & & & & 4 & WM2, 3, 4, 6, \\
\hline Placynthiella dasaea (Stirt.) Tønsberg & 1 & 1 & & & $\mathrm{R}$ & & & 12 & $\begin{array}{l}\text { MC1, 2, 7, MM2, 3, 4, 7, WM7, } \\
\text { CC2, 4, CM5, AM5 }\end{array}$ \\
\hline $\begin{array}{l}\text { Placynthiella icmalea (Ach.) Coppins \& P. } \\
\text { James }\end{array}$ & 1 & 1 & & & $\mathrm{R}$ & & & 16 & $\begin{array}{l}\text { MC2, 5, 7, MM3, 5, 6, 7, PM4, } \\
\text { 5, WM1, 3, CM5, 6, AM5, 6, } 7\end{array}$ \\
\hline $\begin{array}{l}\text { Placynthiella uliginosa (Schrad.) Coppins \& } \\
\text { P. James }\end{array}$ & 1 & & & & $\mathrm{R}$ & & & 3 & PM3, WM6, AM7 \\
\hline $\begin{array}{l}\text { Platismatia glauca (L.) W.L. Culb. \& C.F. } \\
\text { Culb. }\end{array}$ & & & & & $\mathrm{R}$ & & NT & 15 & $\begin{array}{l}\text { MC2, 3, 4, MM1, 3, WM2, 6, } \\
\text { AC3, CC1, 4, 5, 6, CM2, 5, } 6\end{array}$ \\
\hline Porina aenea (Wallr.) Zahlbr. & 1 & & & & $\mathrm{R}$ & & & 4 & MC1, MM1, 6, 7 \\
\hline Porina lectissima (Fr.) Zahlbr. & 1 & & & & & & & 1 & MM1 \\
\hline Pseudephebe pubescens (L.) M. Choisy & & & & & & EN & & 11 & $\begin{array}{l}\mathrm{PM} 2,6, \mathrm{AC} 1,3,4,5,6,7 \\
\mathrm{AM} 1,2,3\end{array}$ \\
\hline Pseudevernia furfuracea (L.) Zopf & & & & & & & NT & 37 & $\begin{array}{l}\text { MC2, 3, 4, 5, 6, 7, MM1, 2, 3, } \\
\text { 5, 6, 7, PM1, 2, 3, 4, 5, 6, WM1, } \\
\text { 2, 3, 4, 5, 6, 7, AC 3, CC1, 2, 3, } \\
\text { 4, 6, 7, CM1, 2, 4, 5, AM1 }\end{array}$ \\
\hline Pycnothelia papillaria (Ehrh.) L.M. Dufour & & & & & EN & $\mathrm{EN}$ & $\mathrm{VU}$ & 1 & AC4 \\
\hline Pyrenula nitida (Weigel) Ach. & & & & & & $\mathrm{VU}$ & EN & 5 & $\mathrm{MC} 1,2,3,4, \mathrm{MM} 2$ \\
\hline
\end{tabular}


Table 3 (continued)

\begin{tabular}{|c|c|c|c|c|c|c|c|c|c|}
\hline Lichen taxa & $\begin{array}{l}\text { New R } \\
\text { Mts }\end{array}$ & $\begin{array}{l}\text { New } \\
\mathrm{RO}\end{array}$ & $\begin{array}{l}\mathrm{RL} \\
\mathrm{RO}\end{array}$ & $\begin{array}{l}\mathrm{RL} \\
\mathrm{UK}\end{array}$ & $\begin{array}{l}\text { RL } \\
\mathrm{HU}\end{array}$ & $\begin{array}{l}\text { RL } \\
\text { PL }\end{array}$ & $\begin{array}{l}\text { RL } \\
\text { SL }\end{array}$ & Obs & Plot codes \\
\hline $\begin{array}{l}\text { Pyrenula nitidella (Flörke ex Schaer.) Müll. } \\
\text { Arg. }\end{array}$ & 1 & & & & $\mathrm{R}$ & EN & CR & 1 & MC3 \\
\hline Ramalina canariensis J. Steiner & 1 & & & $\mathrm{R}$ & & & & 3 & $\mathrm{MC} 2,3,6$ \\
\hline Ramalina farinacea (L.) Ach. & & & & & & $\mathrm{VU}$ & EN & 3 & $\mathrm{MC} 2,4,5$ \\
\hline Ramalina fastigiata (Pers.) Ach. & & & & & & $\mathrm{EN}$ & EN & 1 & MC2 \\
\hline Ramalina pollinaria (Westr.) Ach. & & & & & & $\mathrm{VU}$ & VU & 1 & MC3 \\
\hline Rinodina sophodes (Ach.) A. Massal. & & & & & & EN & VU & 3 & WM2, 3, 5 \\
\hline Ropalospora viridis (Tønsberg) Tønsberg & & & & & & & & 2 & $\mathrm{MC} 2,5$ \\
\hline $\begin{array}{l}\text { Scoliciosporum chlorococcum (Graewe ex } \\
\text { Stenh.) Vèzda }\end{array}$ & 1 & & & & & & & 12 & $\begin{array}{l}\text { MC7, MM2, 4, 5, 7, WM3, 5, 6, } \\
\text { 7, CC7, CM3, } 5\end{array}$ \\
\hline Scoliciosporum sarothamni (Vain.) Vězda & 1 & 1 & & & & & & 7 & MC6, MM3, 5, 7, WM5, 6, 7 \\
\hline Scoliciosporum umbrinum (Ach.) Arnold & & & & & & & & 4 & MM2, 4, 5, WM5, \\
\hline Sphaerophorus fragilis (L.) Pers. & & & & & & EN & VU & 4 & $\mathrm{PM} 2, \mathrm{AC} 3,4,6$ \\
\hline Stereocaulon alpinum Laurer & & & VU & & & EN & VU & 1 & AC3 \\
\hline Strigula jamesii (Swinscow) R.C. Harris & 1 & 1 & & & & & & 1 & MC5 \\
\hline Thamnolia vermicularis (Sw.) Schaer. & & & & VU & & $\mathrm{LC}$ & & 14 & $\begin{array}{l}\text { PM5, 7, AC1, 2, 3, 4, AC5, 6, 7, } \\
\text { AM1, 2, 3, 4, } 5\end{array}$ \\
\hline Thelotrema lepadinum (Ach.) Ach. & 1 & & & & & EN & CR & 2 & $\mathrm{MC} 3,4$ \\
\hline Trapelia corticola Coppins \& P. James & 1 & 1 & & & & & & 1 & PM1 \\
\hline Trapeliopsis flexuosa (Fr.) Coppins \& P. James & & & & & & & & 13 & $\begin{array}{l}\text { MM2, 3, 6, WM5, 6, AC5, 7, } \\
\text { CC 3, 4, 5, 7, CM6, } 7\end{array}$ \\
\hline $\begin{array}{l}\text { Trapeliopsis gelatinosa (Flörke) Coppins \& } \\
\text { P. James }\end{array}$ & 1 & & & & & NT & & 3 & MC1, CC2, AM5 \\
\hline Trapeliopsis granulosa (Hoffm.) Lumbsch & 1 & & & & & & & 22 & $\begin{array}{l}\text { MC7, PM1, 3, 4, 5, 6, AC1, 2, } \\
3,4,5,6,7, \mathrm{CC} 3, \mathrm{CM} 2, \mathrm{AM} 1, \\
2,3,4,5,6,7\end{array}$ \\
\hline $\begin{array}{l}\text { Trapeliopsis pseudogranulosa Coppins \& P. } \\
\text { James }\end{array}$ & 1 & 1 & & & & & & 2 & PM5, CC1 \\
\hline $\begin{array}{l}\text { Trapeliopsis viridescens (Schrad.) Coppins \& } \\
\text { P. James }\end{array}$ & 1 & & & & & NT & & 4 & $\mathrm{MC} 1,7, \mathrm{AC7}, \mathrm{CC} 6$ \\
\hline Tuckermannopsis chlorophylla (Willd.) Hale & & & VU & & $\mathrm{EN}$ & $\mathrm{VU}$ & NT & 2 & MC2, WM1 \\
\hline Umbilicaria crustulosa (Ach.) Lamy & 1 & & & & & EN & NT & 2 & $\mathrm{AC7}, \mathrm{CC} 4$ \\
\hline Umbilicaria cylindrica (L.) Delise & & & & & $\mathrm{RE}$ & & & 28 & $\begin{array}{l}\mathrm{PM} 1,2,3,4,5,6,7, \mathrm{WM} 1,4, \\
6, \mathrm{AC} 1,2,3,4,5,6,7, \mathrm{CC} 2,3 \\
4,5, \mathrm{AM} 1,2,3,4,5,6,7\end{array}$ \\
\hline Umbilicaria deusta (L.) Baumg. & & & & & $\mathrm{CR}$ & LC & & 11 & $\begin{array}{l}\mathrm{AC} 1,2,4,6, \mathrm{CC} 4, \mathrm{AM} 1,2,4 \\
5,6,7\end{array}$ \\
\hline Umbilicaria polyphylla (L.) Baumg. & & & & & CR & LC & & 6 & PM6, WM1, AC6, AM1, 3, 6 \\
\hline Umbilicaria vellea (L.) Ach. & & & & & & EN & VU & 1 & CC4 \\
\hline Usnea chaetophora Stirt. & 1 & 1 & & & & & & 2 & $\mathrm{MC} 2, \mathrm{CC} 4$ \\
\hline Usnea diplotypus Vain. & 1 & 1 & & & $\mathrm{CR}$ & $\mathrm{RE}$ & & 2 & $\mathrm{CC} 4,5$ \\
\hline Usnea filipendula Stirt. & & & & & $\mathrm{CR}$ & $\mathrm{VU}$ & CR & 5 & $\mathrm{MC} 4, \mathrm{CC} 1,3,4,5, \mathrm{CM} 2$, \\
\hline Usnea florida (L.) Weber ex F.H. Wigg. & & & & VU & CR & CR & CR & 1 & MC3 \\
\hline Usnea fulvoreagens (Räsänen) Räsänen & 1 & & $\mathrm{EN}$ & & $\mathrm{CR}$ & CR & $\mathrm{RE}$ & 1 & MC4 \\
\hline Usnea glabrescens (Nyl. ex Vain.) Vain. & 1 & & & & & & & 1 & CC4 \\
\hline Usnea hirta (L.) Weber exF.H. Wigg. & & & & & & VU & VU & 5 & WM2, 3, 4, 5, CC6 \\
\hline Usnea lapponica Vain. & 1 & & & & & & & 1 & MC4 \\
\hline Usnea scabrata $\mathrm{Nyl}$. & 1 & & & & & & & 1 & MM1 \\
\hline Usnea subfloridana Stirt. & & & & & $\mathrm{CR}$ & $\mathrm{EN}$ & CR & 4 & MC2, 4, 6, WM5 \\
\hline Vulpicida pinastri (Scop.) J.-E. Mattsson & 1 & & & & & NT & NT & 11 & $\begin{array}{l}\text { PM4, 6, 7, WM2, 4, 6, 7, CC4, } \\
5,6,7\end{array}$ \\
\hline
\end{tabular}


Table 3 (continued)

\begin{tabular}{|c|c|c|c|c|c|c|c|c|c|}
\hline Lichen taxa & $\begin{array}{c}\text { New R } \\
\text { Mts }\end{array}$ & $\begin{array}{l}\text { New } \\
\text { RO }\end{array}$ & $\begin{array}{l}\text { RL } \\
\text { RO }\end{array}$ & $\begin{array}{l}\text { RL } \\
\text { UK }\end{array}$ & $\begin{array}{l}\mathrm{RL} \\
\mathrm{HU} \\
\end{array}$ & $\begin{array}{l}\text { RL } \\
\text { PL }\end{array}$ & $\begin{array}{l}\text { RL } \\
\text { SL }\end{array}$ & Obs & Plot codes \\
\hline $\begin{array}{l}\text { Xanthoparmelia conspersa (Ehrh. ex Ach.) } \\
\text { Hale }\end{array}$ & & & & & & & & 1 & WM4 \\
\hline Xanthoparmelia somloensis (Gyeln.) Hale & 1 & & & & & & & 1 & WM2 \\
\hline Xanthoria candelaria (L.) Th. Fr. & 1 & & & & & & & 2 & WM4, 7 \\
\hline Xanthoria cf. ulophyllodes ${ }^{*}$ Räsänen & & & & & & $\mathrm{VU}$ & $\mathrm{VU}$ & 1 & WM2 \\
\hline Xanthoria parietina (L.) Beltr. & & & & & & & NT & 5 & WM2, 3, 4, 5, 6 \\
\hline Xanthoria polycarpa (Hoffm.) Rieber & 1 & & & & & & NT & 1 & WM5, 7 \\
\hline Xylographa parallela (Ach.) Fr. & & & & & & $\mathrm{EN}$ & & 3 & MC2, WM4, CC1 \\
\hline Xylographa vitiligo (Ach.) J.R. Laundon & 1 & & & & & DD & $\mathrm{EN}$ & 2 & MM3, PM7 \\
\hline Totals & 182 & 67 & 12 & 8 & 65 & 125 & 96 & & \\
\hline
\end{tabular}

Several inconspicuous taxa (Micarea sp., Placynthiella sp., Scoliciosporum sp., Lichenomphalia umbelifera, Lichenomphalia velutina) possibly overlooked in previous surveys, were found mainly because of the systematic sampling protocol used here, which forced us to consider all lichens present in small sampling plots.

Another group of neglected species in Romania includes taxa that only recently have received a thorough taxonomic treatment that makes identification possible in routine lichen diversity assessments. Among these is the genera Lepraria for which we found 13 species, all new for the Rodnei Mountains, and 10 species new for the country.

The numerous new species for the region is mainly due to the fact that the previous studies focused on rocks, whereas substrates such as trees, soil, and wood, were poorly investigated. For example, there are only 49 previously reported species on soil substrate versus the 108 taxa that we report here. We only confirm 158 out of the 260 species present in the previous checklist, which was represented mainly by saxicolous lichens (ca $57 \%$ of total) (APNMR, 2013), including crustose, foliose and fruticose species, whereas in our survey the crustose saxicolous lichens were not sampled.

We found a relatively high number of species that are restricted to conserved habitats in Rodnei Mountains and which are known as indicators of ancient woodland and ecological continuity in forest landscapes (Rose, 1976; Goward, 1995; Wolseley, 1995; Thor, 1995; Gauslaa \& Solhaug, 1996): Arthonia caesia, Bryoria lanestris, Cetrelia olivetorum, Chaenotheca brachypoda, Ch. brunneola, Heteroder- mia speciosa, Lecanora cinereofusca, Lobaria pulmonaria, Loxospora cismonica, Megalospora tuberculosa, Menegazzia terebrata, Pertusaria coccodes, Thelotrema lepadinum, Usnea florida and $U$. subfloridana.

Also species from Red Lists, which depend on rare and often threatened habitats (Thor, 1995), such as Anisomeridium biforme and Usnea fulvoreagens, are extinct in some regions of the Carpathians (Pisút et al., 2001) but were recorded (once each) in conserved mixed forests in the present study of the Rodnei Mountains. Other examples of species limited to conserved areas, having Critically Endangered status in the Red Lists and being recorded once or twice in our study include: Arthonia vinosa, Bellemerea cinereorufescens, Bryoria capillaris, B. chalibeiformis, Cetraria aculeata, Cladonia magyarica, Hypogymnia vittata, Icmadophila ericetorum, Lecanactis abietina, Lecanora albella, Mycoblastus sanguinarius, Nephroma parile, Phaeophyscia endophoenicea and Pyrenula nitidella (Table 3). All these species stress the importance of maintaining their suitable habitats, which are currently restricted to protected areas.

The present study, with its large number of new species for Romanian lichen flora and for the Rodnei Mountains region, suggests the need of more detailed inventories. To this day, there is no official Red List of lichens for Romania, except a manuscript dealing with macrolichens only (Bartok \& Crișan, personal communication). In this context lichen diversity and richness inventories are important for better estimating the degree of threat for each species. This will enable the compilation of a comprehensive Red List of Lichens from Romania that will be an invalu- 
able tool for promoting lichen conservation in the country (Thor, 1995; Dietrich \& Scheidegger, 1997; Scheidegger et al., 2000).

The high total number of lichen species, the large number of species that appear in Red Lists, together with the indicator species for ancient woodlands and ecological continuity of forest landscapes, underline the high value of conserved habitats in the Rodnei Mountains. The existing National Park here has the potential of conserving a considerable part of the lichen diversity and the lichen's characteristic habitats. Their presence is also important for other groups of organisms, which depend on lichens or their habitats, thus emphasizing the biocomplexity of microbial and invertebrate communities. It is important that at least these „islands” such as Rodnei Mountains to be conserved if otherwise natural resources are still exploited in a non-sustainable manner that is leading to a substantial loss of biodiversity.

\section{ACKNOWLEDGEMENTS}

This work was possible with the financial support of the Sectoral Operational Programme for Human Resources Development 2007-2013, co-financed by the European Social Fund, under the project number POSDRU/107/1.5/S/76841 with the title „Modern Doctoral Studies: Internationalization and Interdisciplinarity". We also acknowledge financial support from the Federal Office for the Environment (FOEN) to Christoph Scheidegger.

\section{REFERENCES}

APNMR (Rodnei Mountains National Park Administration). Management Plan 2013 Rodnei Mountains National Park, Biosphere Reserve (NATURA 2000 SAC and SPA) (In Romanian). http://www.parcrodna.ro/.

Bartók, K. 1999. Pesticide usage and epiphytic lichen diversity in Romanian orchards. The Lichenologist 31(1): 21-25.

Cieslinski, S., Czyzewska K. \& Fabiszewski, J. 2003. Czerwona lista porostów wymarlych i zagrozonych w Polsce. Red List of extinct and threatened lichens in Poland. - In: Czyzewska, K. (ed.). Zagrozenie Porostów w Polsce. The threat to lichens in Poland. Monographiae Botanicae 91: 13-49.

Ciurchea, M. 2004. Key to Lichens from Romania (In Romanian). Ed. Bit, Iași. 471 pp.

Çobanoğlu, G., Yavuz, M., Costache, I. \& Radu, I. 2011. Additional and new lichen records from
Cozia National Park, Romania. Mycotaxon 114: 193-196. http://dx.doi.org/10.5248/114.193

Çobanoğlu, G., Yavuz, M., Costache, I., Radu, I., Açikgöz, B. \& Baloniu, L. 2009. Epiphytic and terricolous lichens diversity in Cozia National Park (Romania). Muzeul Olteniei Craiova. Oltenia. Studii şi comunicări. Ştiinţele Naturii 25: 17-22.

Crișan, F. 2006. Five new species for the Romanian lichen flora. In: Gafta, D. \& Akeroyd, J. (eds). Nature Conservation, Concepts and Practice. Series: Environmental Science and Engineering, pp. 131-136.

Didukh, Y. A. (ed.) 2009. Red data book of Ukraine, Vegetable kingdom (In Ukrainian). Globalconsalting. $912 \mathrm{pp}$.

Dietrich , M. \& Scheidegger, C. 1997. A representative survey of frequency of epiphytic lichens at the regional and national levels and its use for the Red List of Switzerland. In: Türk, R. \& Zorrer, R. (eds). Progress and Problems in Lichenology in the Nineties - IAL3. Bibliotheca Lichenologica 68: $145-154$.

Gauslaa, Y. \& Solhaug, K. A. 1996. Differences in the susceptibility to light stress between epiphytic lichens of ancient and young boreal forest stands. Functional Ecology 10(3): 344-354. http: / / dx.doi. org/ $10.2307 / 2390282$

Gorduza V. 1983. Physico-geographical caracterisation of the Pietrosu Rodnei Nature Reserve. In: Pietrosul Rodnei at 50 years (In Romanian). Academia RSR Cluj Napoca-Baia Mare, pp: 56-66.

Goward, T. 1995. Nephroma occultum and the maintenance of lichen diversity in British Columbia. Mitteilungen der Eidgenössischen Forschungsanstalt für Wald, Schnee und Landschaft 70: 11-27.

Ignatov, M.S., Ignatova, E.A., Konstantinova, N. A., Pronkina, G. A. \& Urbanavichus, G. P. 2004. Lichens / The present-day state of biological diversity within protected areas. Issue 3. Lichens and Bryophytes. Moscow, pp 6-7.

Lackovičová, A. \& Guttová, A. 2006. Lichen diversityhistory, contemporary occurrence and trends in Slovakia: Gyalecta ulmi and Leptogium saturninum. In: Lackovičová, A., Guttová, A., Lisická, E. \& Lizoň, P. (eds). Central European Lichens Diversity and Threat. Mycotaxon Ltd., Ithaca, and Institute of Botany, Slovak Academy of Sciences, Bratislava, pp. 219-240.

Lőkös, L. \& Tóth, E. 1996. Red List of lichens of Hungary (a proposal). Vegetation Mapping and Botanical Research On Nature Conservation Purpose 7: 337-343.

Nascimbene, J., Thor, G. \& Nimis, P. L. 2013. Effects of forest management on epiphytic lichens in temperate deciduous forests of Europe-A review. Forest Ecology and Management 298: 27-38. http://dx.doi.org/10.1016/j.foreco.2013.03.008

Pisút, I., Guttová, A., Lackovicová, A. \& Lisická, E. 2001. Cerveny zoznam lisajníkov Slovenska (December 2001) [Red List of lichens of Slovakia 
(December 2001)] Ochrana Prírody, Supplement 20: 23-30.

Rose, F. 1976. Lichenological indicators of age and environment continuity in woodlands. In: Brown, D. H., Hawksworth, D. L. \& Bailey, R. H. (eds). Lichenology: Progress and Problems. Academic Press, London, pp. 279-307.

Scheidegger, C. 1985. Systematische Studien zur Krustenflechte Anzina carneonivea (Trapeliaceae, Lecanorales). Nova Hedwigia 41:191-218.

Scheidegger, C., Groner, U., Keller, C. \& Stofer, S. 2002. Biodiversity Assessment Tools - Lichens. In: Nimis, P.L., Scheidegger, C. \& Wolseley, P.A. (eds.). Monitoring with Lichens - Monitoring Lichens. Dordrecht, Boston, London, Kluwer Academic, pp. 359-365. http://dx.doi.org/10.1007/97894-010-0423-7_35

Scheidegger, C., Stofer, S., Dietrich, M., Groner, U., Keller, C. \& Roth, I. 2000. Estimating regional extinction probabilities and reduction in populations of rare epiphytic lichen-forming fungi. Forest Snow and Landscape Research 75(3): 415-433.

Scheidegger, C. \& Werth, S. 2009. Conservation strategies for lichens: insights from population biology. Fungal biology reviews 23: 55-66. http://dx.doi. org/10.1016/j.fbr.2009.10.003

Smith, C. W., Aproot, A., Coppins, B. J., Fletcher, A., Gilbert, O. L., James, P. W. \& Wolseley, P. A. 2009. The Lichens of Great Britain and Ireland. Ed. British Lichen Society, London. 1046 pp.

Thor, G. 1995. Red Lists - Aspects of their compilation and use in Lichen conservation. Mitteilungen der Eidgenössischen Forschungsanstalt für Wald, Schnee und Landschaft 70: 11-27.

Tønsberg. T. 1992. The sorediate and isidiate, corticolous, crustose lichens in Norway. Sommerfeltia 14: $1-331$.

Vondrák, J. \& Šoun, J. 2008.Some newly recorded and noteworthy lichen-forming and lichenicolous fungi from Romania. Acta Botanica Hungarica 50(1-2): 215-221. http://dx.doi.org/10.1556/ ABot.50.2008.1-2.16

White, F. J. \& James, P. W. 1985. A new guide to microchemical theniques for the identification of lichen substances. British Lichen Society Bulletin Supplement. 27 pp.

Wirth, V. 1995. Die Flechten Baden-Württembergs. (2 vols) Ed. Ulmer, Stuttgart. 1006 pp.

Wolseley, P. A. 1995. A global perspective on the status of lichens and their conservation. Mitteilungen der Eidgenössischen Forschungsanstalt für Wald, Schnee und Landschaft 70: 11-27.

Yavuz, M. \& Çobanolu, G., 2008. Lichen records from Dobrogea, Romania. Muzeul Olteniei Craiova. Oltenia. Studiiși comunicări. Științele Naturii 24: 17-21.

Zoller, S., Frey, B. \& Scheidegger, C., 2000. Juvenile development and diaspore survival in the threatened epiphytic lichen species Sticta fuliginosa, Leptogium saturninum and Menegazzia terebrata: conclusions for in situ conservation. Plant Biology 2: 496-504. http://dx.doi. org/ 10.1055/s-2000-5954 
116 Folia Cryptog. Estonica 